\title{
Energy Harvesting Cooperative Diamond Channel
}

\author{
Berk Gurakan \\ Sennur Ulukus \\ Department of Electrical and Computer Engineering \\ University of Maryland, College Park, MD 20742 \\ gurakan@umd.edu \\ ulukus@umd.edu
}

\begin{abstract}
We consider the energy harvesting diamond channel, where the source and two relays harvest energy from nature. The physical layer is modeled as a concatenation of a broadcast and a multiple access channel. We find the optimal offline transmit power and rate allocations that maximize the end-toend throughput. First, we show that there exists an optimal source power allocation which is equal to the single-user optimal power allocation for the source energy arrivals and does not depend on the relay energy arrivals. Second, we show that the fraction of the power spent on each broadcast link depends on the energy arrivals for the relays. Specifically, we show that the optimal source rate allocation can be found by solving an optimal broadcasting problem with slot-dependent user priorities and these priorities can change only at instants where one of the relay data buffers is empty. Finally, we decompose the problem into inner and outer optimization problems and solve the overall problem by iterating between the two.
\end{abstract}

\section{INTRODUCTION}

We consider the cooperative energy harvesting diamond channel [1], see Fig. 1, where all transmitters harvest energy from nature. We model the physical layer as a concatenation of a Gaussian broadcast channel and a Gaussian multiple access channel. Since the broadcast channel is degraded, one of the relays has the message of the other relay. Therefore, the multiple access channel is an extended multiple access channel with common data [2]. Our aim is to determine the optimum power and rate allocation policies of the users in order to maximize the end-to-end throughput of this system.

There has been a considerable amount of recent work in power control for energy harvesting communications [3]-[19]. In [3], the transmission completion time minimization problem is solved for an unlimited-sized battery. In [4], the throughput maximization problem is solved and its equivalence to the transmission completion time minimization problem is shown for an arbitrarily sized battery. In [5]-[10] the problem is extended to fading, broadcast, multiple access and interference channels. Throughput maximization problem with battery imperfections is considered in [11], [12] and processing costs are incorporated in [13], [14]. Of particular relevance are [15]-[19], where two-hop communication is considered with energy harvesting nodes for half- or full-duplex relay settings. Recently, in [20], a two-hop communication system with two parallel relays, with and without energy cooperation is studied. In [20], all four links of the broadcast and multiple access channels were restricted to be orthogonal, and no

This work was supported by NSF Grants CNS 13-14733, CCF 14-22111 and CCF 14-22129.

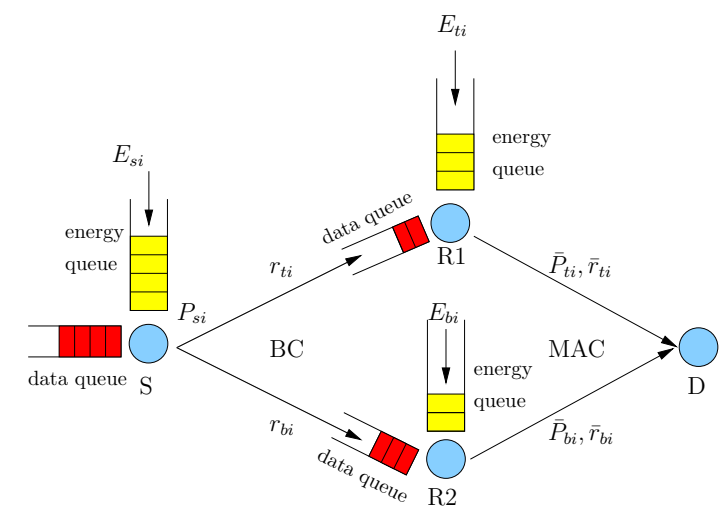

Fig. 1. Cooperative diamond network with energy harvesting nodes.

storage of data was allowed at the relays due to strict delay constraints. The setting in the current paper can be viewed as a generalization of [20] to general broadcast and multiple access channels, and general data storage at the relays.

In this paper, we first show that there exists an optimal source power allocation policy which is equal to the singleuser optimal power policy for the source energy arrivals and does not depend on the relay energy arrivals. This is a generalization of [6], [8], which proved the optimality of a single-user power allocation for the capacity region of a broadcast channel; our work shows that the result remains the same even when the broadcast channel is concatenated with a multiple access channel. Our result is also a generalization of the separation result proved in [15], [16], which showed that, in a single relay channel, the source can optimize its transmit power irrespective of relay's energy arrivals; our work shows that this result remains the same for the case of two relays forming a multiple access second hop. Next, we show that even though the total power can be selected as the singleuser optimal power, the fraction of the power spent on each broadcast link depends on the energy arrivals of the relays. Specifically, we show that the optimal source rate allocation can be found by solving an optimal broadcasting problem with slot-dependent user priorities and these priorities can change only at instants when one of the relay data buffers is empty. Finally, we decompose the problem into inner and outer optimization problems and solve the problem iteratively.

\section{SYSTEM MODEL}

We consider the energy harvesting diamond channel shown in Fig. 1. The harvested energies are saved in the correspond- 
ing batteries. The physical layer is modeled as a concatenation of a broadcast channel and a multiple access channel. In the broadcast channel, relay 1 is the stronger receiver: the channel noises have variances $\sigma_{1}^{2} \leq \sigma_{2}^{2}$. The Gaussian broadcast channel capacity region with transmitter power $P$ is given by

$$
\mathcal{C}_{B C}(P)=\left\{r_{1} \leq f\left(\frac{\alpha P}{\sigma_{1}^{2}}\right), \quad r_{2} \leq f\left(\frac{(1-\alpha) P}{\alpha P+\sigma_{2}^{2}}\right)\right\}
$$

where $\alpha$ is the fraction of power spent for the message of user 1 , and $f(x) \triangleq \frac{1}{2} \log (1+x)$. The function $g\left(r_{1}, r_{2}\right)$ is the minimum energy required to transmit at rates $\left(r_{1}, r_{2}\right)$ :

$$
g\left(r_{1}, r_{2}\right) \triangleq \sigma_{1}^{2} 2^{2\left(r_{1}+r_{2}\right)}+\left(\sigma_{2}^{2}-\sigma_{1}^{2}\right) 2^{2 r_{2}}-\sigma_{2}^{2}
$$

and is strictly convex in $\left(r_{1}, r_{2}\right)$. Since relay 2 is degraded with respect to relay 1 , relay 1 can decode the messages intended for relay 2. Therefore, the second hop is an extended multiple access channel with common data. The capacity region for this channel with transmitter powers $\left(P_{1}, P_{2}\right)$ and Gaussian noise power $\sigma_{3}^{2}$ is given as [1], [2]:

$$
\begin{aligned}
& \mathcal{C}_{E M A C}\left(P_{1}, P_{2}\right)=\left\{r_{1} \leq f\left((1-\beta) P_{1} / \sigma_{3}^{2}\right),\right. \\
&\left.r_{1}+r_{2} \leq f\left(\left(P_{1}+P_{2}+2 \sqrt{\beta P_{1} P_{2}}\right) / \sigma_{3}^{2}\right)\right\}
\end{aligned}
$$

There are $N$ equal length slots. We refer to relay 1 as the top and relay 2 as the bottom relay and use subscripts $t$ and $b$ to denote their parameters; subscript $s$ denotes source node's parameters. In slot $i$, the source, top and bottom relays harvest energy with amounts $E_{s i}, E_{t i}, E_{b i}$, respectively. We denote the transmission power of the source as $P_{s i}$ and source rates to the top (bottom) relay as $r_{t i}\left(r_{b i}\right)$, the transmission power of the top (bottom) relay to the destination as $\bar{P}_{t i}\left(\bar{P}_{b i}\right)$ and data rates of the top (bottom) relays to the destination as $\bar{r}_{t i}\left(\bar{r}_{b i}\right)$. We denote these power and rate sequences with the vectors $\mathbf{P}_{s}, \overline{\mathbf{P}}_{t}, \overline{\mathbf{P}}_{b}, \mathbf{r}_{t}, \mathbf{r}_{b}, \overline{\mathbf{r}}_{t}, \overline{\mathbf{r}}_{b}$. The energy that has not arrived yet cannot be used, leading to the following energy causality constraints at all transmitters:

$$
\begin{aligned}
& \sum_{i=1}^{k} \bar{P}_{t i} \leq \sum_{i=1}^{k} E_{t i}, \quad \forall k \\
& \sum_{i=1}^{k} \bar{P}_{b i} \leq \sum_{i=1}^{k} E_{b i}, \quad \forall k \\
& \sum_{i=1}^{k} P_{s i} \leq \sum_{i=1}^{k} E_{s i}, \quad \forall k
\end{aligned}
$$

The relays cannot forward data that has not arrived yet, leading to the following data causality constraints at the relays:

$$
\begin{aligned}
& \sum_{i=1}^{k} \bar{r}_{t i} \leq \sum_{i=1}^{k} r_{t i}, \quad \forall k \\
& \sum_{i=1}^{k} \bar{r}_{b i} \leq \sum_{i=1}^{k} r_{b i}, \quad \forall k
\end{aligned}
$$

The rate allocations must be achievable for each channel:

$$
\left(r_{t i}, r_{b i}\right) \in \mathcal{C}_{B C}\left(P_{s i}\right), \quad \forall i
$$

$$
\left(\bar{r}_{t i}, \bar{r}_{b i}\right) \in \mathcal{C}_{E M A C}\left(\bar{P}_{t i}, \bar{P}_{b i}\right), \quad \forall i
$$

We aim to maximize the end-to-end throughput:

$$
\max _{\mathbf{P}_{s}, \overline{\mathbf{P}}_{t}, \overline{\mathbf{P}}_{b}, \mathbf{r}_{t}, \mathbf{r}_{b}, \overline{\mathbf{r}}_{t}, \overline{\mathbf{r}}_{b}} \quad \sum_{i=1}^{N} \bar{r}_{t i}+\bar{r}_{b i}
$$

\section{Necessary Conditions And Optimal Profile}

We first prove some properties of the optimal solution.

Lemma 1 Either the source energy or both of the relay energies must be consumed fully.

Proof: The proof follows by contradiction. If any excess energy is left then we can increase the rates, which contradicts optimality.

Lemma 2 There exists an optimal source profile $\left(\mathbf{P}_{s}^{*}, \mathbf{r}_{t}^{*}, \mathbf{r}_{b}^{*}\right)$ that is on the boundary of the broadcast capacity region in each slot, i.e., $r_{t i}^{*}=f\left(\frac{\alpha_{i} P_{s i}^{*}}{\sigma_{1}^{2}}\right), r_{b i}^{*}=f\left(\frac{\left(1-\alpha_{i}\right) P_{s i}^{*}}{\alpha P_{s i}^{*}+\sigma_{2}^{2}}\right), \forall i$.

Proof: In slots where these constraints are satisfied with strict inequality, we can increase $r_{t i}^{*}$ or $r_{b i}^{*}$ without violating any feasibility constraints as we can always increase the right hand sides of the data feasibility constraints in (7) and (8).

Using Lemma 2 we can remove the broadcast capacity region constraints from the problem and let $P_{s i}=g\left(r_{t i}, r_{b i}\right)$. The corresponding energy causality constraints for the source node can now be written as:

$$
\sum_{i=1}^{k} g\left(r_{t i}, r_{b i}\right) \leq \sum_{i=1}^{k} E_{s i}, \quad \forall k
$$

The optimization problem can now be written as:

$$
\begin{aligned}
& \max _{\overline{\mathbf{P}}_{t}, \overline{\mathbf{P}}_{b}, \mathbf{r}_{t}, \mathbf{r}_{b}, \overline{\mathbf{r}}_{t}, \overline{\mathbf{r}}_{b}} \sum_{i=1}^{N} \bar{r}_{t i}+\bar{r}_{b i} \\
& \text { s.t. (4)-(5), (7)-(8), (10), (12) }
\end{aligned}
$$

Now, we have a theorem which states a key structural property of the optimal policy and is proved in the Appendix.

Theorem 1 There exists an optimal total source power sequence $g\left(r_{t i}^{*}, r_{b i}^{*}\right)$ which is the same as the single-user optimal transmit power sequence for the energy arrivals $E_{s i}$.

Theorem 1 tells us that there exists a solution to the problem in (13) in which $g\left(r_{t i}^{*}, r_{b i}^{*}\right)=P_{i}$, where $P_{i}$ are the singleuser optimal transmit powers for the energy arrivals $E_{s i}$. This constraint can always be relaxed to $g\left(r_{t i}, r_{b i}\right) \leq P_{i}$. Using Theorem 1, the optimization problem becomes:

$$
\begin{aligned}
& \max _{\overline{\mathbf{P}}_{t}, \overline{\mathbf{P}}_{b}, \mathbf{r}_{t}, \mathbf{r}_{b}, \overline{\mathbf{r}}_{t}, \overline{\mathbf{r}}_{b}} \sum_{i=1}^{N} \bar{r}_{t i}+\bar{r}_{b i} \\
& \text { s.t. (4)-(5), (7)-(8), (10), } g\left(r_{t i}, r_{b i}\right) \leq P_{i}
\end{aligned}
$$


Theorem 1 generalizes the results of [6], [8] to the case of concatenated networks, and the results of [15], [16] to the case of multiple relays. While the source power does not depend on the energy arrival profile of the relays, the fraction of the total power spent on each broadcast link depends on the energy arrival profile of the relays. In the following lemmas, we show how to find the distribution of power over the broadcast links.

Lemma 3 There exists a positive real vector $\boldsymbol{\mu} \triangleq$ $\left\{\mu_{i}\right\}_{i=1}^{N}, \mu_{i} \in \mathbb{R}^{+}$such that $\left(r_{t i}^{*}, r_{b i}^{*}\right)$ simultaneously solves the problem in (14) and the following optimization problem:

$$
\begin{aligned}
\max _{r_{t i}, r_{b i}} & \sum_{i=1}^{N} \mu_{i} r_{t i}+\sum_{i=1}^{N} r_{b i} \\
\text { s.t. } & g\left(r_{t i}, r_{b i}\right) \leq P_{i}
\end{aligned}
$$

Lemma $4 \mu_{i}$ can increase (decrease) only when the bottom (top) data buffer is empty.

The proof of Lemma 3 follows from the convexity of the broadcast capacity region and Lemma 2; and the proof of Lemma 4 follows from the correspondence between the Lagrange multipliers of the problems in (14) and (15).

In a single-hop broadcasting problem as in [6]-[8], the user priorities are constant in time. Lemmas 3 and 4 show us that the existence of a multiple access layer affects the broadcast layer by introducing variable user priorities in time. The user priorities can change only when one of the data buffers is empty: the priority of the first user can increase only when the bottom data buffer is empty, and can decrease only when the top data buffer is empty. From [6], the solution to (15) is:

$$
\begin{aligned}
& r_{t i}=\frac{1}{2} \log \left(1+\min \left\{P_{c i}, P_{i}\right\}\right) \\
& r_{b i}=\frac{1}{2} \log \left(1+\frac{\left(P_{i}-P_{c i}\right)^{+}}{P_{c i}+\sigma_{2}^{2}}\right)
\end{aligned}
$$

where if $\mu_{i} \geq 1$ all of the power is allocated to the top relay only. If $\mu_{i}<1$, we define

$$
P_{c i} \triangleq\left(\frac{\mu_{i} \sigma_{2}^{2}-\sigma_{1}^{2}}{1-\mu_{i}}\right)^{+}
$$

In other words, given $\left(\mu_{i}, P_{i}\right)$, the rate pairs $\left(r_{t i}, r_{b i}\right)$ can uniquely be determined from (16) and (17). We denote the unique rate pairs found from (16) and (17) for fixed $\left(\mu_{i}, P_{i}\right)$ as $r_{t i}\left(\mu_{i}, P_{i}\right)$ and $r_{b i}\left(\mu_{i}, P_{i}\right)$. Let us define the function $z(\boldsymbol{\mu})$ which is a maximization over $\left(\overline{\mathbf{P}}_{t}, \overline{\mathbf{P}}_{b}, \overline{\mathbf{r}}_{t}, \overline{\mathbf{r}}_{b}\right)$ for fixed $\boldsymbol{\mu}$ :

$$
\begin{aligned}
z(\boldsymbol{\mu})=\max _{\overline{\mathbf{P}}_{t}, \overline{\mathbf{P}}_{b}, \overline{\mathbf{r}}_{t}, \overline{\mathbf{r}}_{b}} \quad & \sum_{i=1}^{N} \bar{r}_{t i}+\bar{r}_{b i} \\
\text { s.t. } \quad & \sum_{i=1}^{k} \bar{r}_{t i} \leq \sum_{i=1}^{k} r_{t i}\left(\mu_{i}, P_{i}\right), \quad \forall k \\
& \sum_{i=1}^{k} \bar{r}_{b i} \leq \sum_{i=1}^{k} r_{b i}\left(\mu_{i}, P_{i}\right), \quad \forall k
\end{aligned}
$$$$
\text { (4)-(5), (10) }
$$

Then, the original problem in (11) is equivalent to:

$$
\max _{\mu_{i} \in[0,1]} z(\boldsymbol{\mu})
$$

We solve the problem in (20) by separately solving the outer and inner maximization problems.

\section{A. Inner Maximization}

In this section, we focus on the inner problem in (19) for fixed $\boldsymbol{\mu}$. This problem is equivalent to a multiple access channel with data arrivals. We define the new variables $\bar{P}_{1 t i}=\left(1-\beta_{i}\right) \bar{P}_{t i}$ and $\bar{P}_{2 t i}=\beta_{i} \bar{P}_{t i}$ and rewrite (19) as:

$$
\begin{aligned}
\max & \sum_{i=1}^{N} \bar{r}_{t i}+\bar{r}_{b i} \\
\text { s.t. } & \sum_{i=1}^{k} \bar{r}_{t i} \leq \sum_{i=1}^{k} r_{t i}\left(\mu_{i}, P_{i}\right), \sum_{i=1}^{k} \bar{r}_{b i} \leq \sum_{i=1}^{k} r_{b i}\left(\mu_{i}, P_{i}\right) \\
& \sum_{i=1}^{k} \bar{P}_{1 t i}+\bar{P}_{2 t i} \leq \sum_{i=1}^{k} E_{t i}, \sum_{i=1}^{k} \bar{P}_{b i} \leq \sum_{i=1}^{k} E_{b i}, \\
& \bar{r}_{t i} \leq f\left(\bar{P}_{1 t i} / \sigma_{3}^{2}\right) \\
& \bar{r}_{t i}+\bar{r}_{b i} \leq f\left(\left(\bar{P}_{1 t i}+\bar{P}_{2 t i}+\bar{P}_{b i}+2 \sqrt{\bar{P}_{2 t i} \bar{P}_{b i}}\right) / \sigma_{3}^{2}\right)
\end{aligned}
$$

We denote the vector triple $\mathcal{P}=\left(\overline{\mathbf{P}}_{1 t}, \overline{\mathbf{P}}_{2 t}, \overline{\mathbf{P}}_{b}\right)$ and define the function $y(\mathcal{P})$ as maximization over $\left(\overline{\mathbf{r}}_{t}, \overline{\mathbf{r}}_{b}\right)$ for fixed $\mathcal{P}$ :

$$
\begin{array}{ll}
y(\mathcal{P}) \triangleq \max _{\left(\overline{\mathbf{r}}_{t}, \overline{\mathbf{r}}_{b}\right)} \sum_{i=1}^{N} \bar{r}_{t i}+\bar{r}_{b i} \\
\text { s.t. } & \sum_{i=1}^{k} \bar{r}_{t i} \leq \sum_{i=1}^{k} r_{t i}\left(\mu_{i}, P_{i}\right), \sum_{i=1}^{k} \bar{r}_{b i} \leq \sum_{i=1}^{k} r_{b i}\left(\mu_{i}, P_{i}\right) \\
& \bar{r}_{t i} \leq f\left(\bar{P}_{1 t i} / \sigma_{3}^{2}\right) \\
& \bar{r}_{t i}+\bar{r}_{b i} \leq f\left(\left(\bar{P}_{1 t i}+\bar{P}_{2 t i}+\bar{P}_{b i}+2 \sqrt{\bar{P}_{2 t i} \bar{P}_{b i}}\right) / \sigma_{3}^{2}\right)
\end{array}
$$

For fixed $\mathcal{P},(22)$ is a linear program, and $y(\mathcal{P})$ can be determined efficiently. We next note the following fact.

Lemma $5 y(\mathcal{P})$ is non-decreasing and concave in $\mathcal{P}$.

Proof: Since increasing the powers can only expand the feasible region, $y$ is non-decreasing in its arguments. To prove the concavity: Let $\mathcal{P}=\left(\overline{\mathbf{P}}_{1 t}, \overline{\mathbf{P}}_{2 t}, \overline{\mathbf{P}}_{b}\right)$ and $\mathcal{Q}=\left(\overline{\mathbf{Q}}_{1 t}, \overline{\mathbf{Q}}_{2 t}, \overline{\mathbf{Q}}_{b}\right)$ be two power vectors. Let $\lambda=1-\bar{\lambda} \in[0,1]$. Let $\left(\overline{\mathbf{r}}_{t}, \overline{\mathbf{r}}_{b}\right)$ solve $y(\mathcal{P})$ and $\left(\overline{\mathbf{s}}_{t}, \overline{\mathbf{s}}_{b}\right)$ solve $y(\mathcal{Q})$. Now, we show that $\left(\lambda \overline{\mathbf{r}}_{t}+\bar{\lambda} \overline{\mathbf{s}}_{t}, \lambda \overline{\mathbf{r}}_{b}+\bar{\lambda} \overline{\mathbf{s}}_{b}\right)$ is feasible for the problem $y(\lambda \mathcal{P}+\bar{\lambda} \mathcal{Q})$. The first two constraints in (22) are linear, thus, their linear combinations are feasible. The third constraint is convex because $f$ is concave. The last constraint is convex because $f$ is concave, non-decreasing, and $\sqrt{\bar{P}_{2 t i} \bar{P}_{b i}}$ is concave. Thus, $\left(\lambda \overline{\mathbf{r}}_{t}+\bar{\lambda} \overline{\mathbf{s}}_{t}, \lambda \overline{\mathbf{r}}_{b}+\bar{\lambda} \overline{\mathbf{s}}_{b}\right)$ is feasible for $y(\lambda \mathcal{P}+\bar{\lambda} \mathcal{Q})$. Now,

$$
y(\lambda \mathcal{P}+\bar{\lambda} \mathcal{Q}) \geq \sum_{i=1}^{N} \lambda \bar{r}_{t i}+\bar{\lambda} \bar{s}_{t i}+\lambda \bar{r}_{b i}+\bar{\lambda} \bar{s}_{b i}
$$




$$
=\lambda y(\mathcal{P})+\bar{\lambda} y(\mathcal{Q})
$$

where (23) follows because the maximum value of the problem can be no smaller than the objective value of any feasible point, and (24) follows from the fact that $\left(\overline{\mathbf{r}}_{t}, \overline{\mathbf{r}}_{b}\right)$ solves $y(\mathcal{P})$ and $\left(\overline{\mathbf{s}}_{t}, \overline{\mathbf{s}}_{b}\right)$ solves $y(\mathcal{Q})$.

The problem in (21) can equivalently be written as:

$$
\begin{array}{rl}
\max _{\overline{\mathbf{P}}_{1 t}, \overline{\mathbf{P}}_{2 t}, \overline{\mathbf{P}}_{b}} & y\left(\overline{\mathbf{P}}_{1 t}, \overline{\mathbf{P}}_{2 t}, \overline{\mathbf{P}}_{b}\right) \\
\text { s.t. } & \sum_{i=1}^{k} \bar{P}_{1 t i}+\bar{P}_{2 t i} \leq \sum_{i=1}^{k} E_{t i}, \quad \forall k \\
& \sum_{i=1}^{k} \bar{P}_{b i} \leq \sum_{i=1}^{k} E_{b i}, \quad \forall k
\end{array}
$$

The problem in (25) is convex as in involves maximizing a concave function over a feasible set with linear constraints. This can be performed efficiently by iterating over feasible $\left(\overline{\mathbf{P}}_{1 t}, \overline{\mathbf{P}}_{2 t}, \overline{\mathbf{P}}_{b}\right)$ such that every iteration increases the objective function, for example, using the method described in [20, Section III.B]. Due to convexity, the convergence to an optimal solution is guaranteed. Once $\left(\overline{\mathbf{P}}_{1 t}^{*}, \overline{\mathbf{P}}_{2 t}^{*}, \overline{\mathbf{P}}_{b}^{*}\right)$ is found, $z(\boldsymbol{\mu})=y\left(\overline{\mathbf{P}}_{1 t}^{*}, \overline{\mathbf{P}}_{2 t}^{*}, \overline{\mathbf{P}}_{b}^{*}\right)$.

\section{B. Outer Maximization}

The outer maximization problem is the problem of finding the optimal $\boldsymbol{\mu}$ in (20). For this purpose, we use the block coordinate descent method on the vector $\boldsymbol{\mu}$. First, we fix $\left(\mu_{1}, \ldots, \mu_{N-1}\right)$ and solve the following problem

$$
\max _{\mu_{N} \in[0,1]} z\left(\mu_{1}, \mu_{2}, \ldots, \mu_{N-1}, \mu_{N}\right)
$$

which can be done using a one-dimensional search on $\mu_{N} \in[0,1]$. Then, using this newly found $\mu_{N}$, we fix $\left(\mu_{1}, \ldots, \mu_{N-2}, \mu_{N}\right)$ and maximize over $\mu_{N-1}$. We cyclically iterate through each $\mu_{i}$, one at a time, maximizing the objective function with respect to that $\mu_{i}$. By construction, the iterations $z\left(\boldsymbol{\mu}^{(k)}\right)$ is a monotone increasing sequence and is bounded because the optimal value of problem (11) is bounded, which guarantees convergence. The iterations converge to an optimal point due to the convexity of the original problem. We can utilize Lemma 4 to search over $\boldsymbol{\mu}$ space more efficiently.

\section{CONCLUSION}

We considered the energy harvesting cooperative diamond channel where the physical layer is modeled as a concatenation of a broadcast channel and a multiple access channel. First, we showed that there exists an optimal source power allocation which is equal to the single-user optimal power policy for the source energy arrivals that does not depend on the relay energy arrivals. Second, we showed that the optimal fractions of the total power can be found by solving an optimal broadcasting problem with slot-dependent user priorities, and these priorities can change only at instants where one of the data buffers is empty. Finally, we decomposed the problem into inner and outer optimization problems and solved the overall problem in an iterative fashion.

\section{Appendix: Proof of Theorem 1}

In this proof, we are only interested in $\left(r_{t i}^{*}, r_{b i}^{*}\right)$. Therefore, to find the necessary optimality conditions, we write the Lagrangian function of the problem in (13) as:

$$
\begin{aligned}
\mathcal{L}= & -\sum_{i=1}^{N} \bar{r}_{t i}+\bar{r}_{b i}+\sum_{k=1}^{N} \lambda_{1 k}\left(\sum_{i=1}^{k} \bar{r}_{t i}-\sum_{i=1}^{k} r_{t i}\right) \\
& +\sum_{k=1}^{N} \lambda_{2 k}\left(\sum_{i=1}^{k} \bar{r}_{b i}-\sum_{i=1}^{k} r_{b i}\right) \\
& +\sum_{k=1}^{N} \gamma_{k}\left(\sum_{i=1}^{k} g\left(r_{t i}, r_{b i}\right)-\sum_{i=1}^{k} E_{s i}\right) \\
& -\sum_{i=1}^{N} \theta_{1 i} r_{t i}-\sum_{i=1}^{N} \theta_{2 i} r_{b i}+\text { other terms }
\end{aligned}
$$

where other terms include the Lagrange multipliers for the other constraints but they are not needed in the proof and are omitted for the sake of brevity. The complementary slackness conditions for these Lagrange multipliers are:

$$
\begin{aligned}
& \lambda_{1 k}\left(\sum_{i=1}^{k} \bar{r}_{t i}-\sum_{i=1}^{k} r_{t i}\right)=\lambda_{2 k}\left(\sum_{i=1}^{k} \bar{r}_{b i}-\sum_{i=1}^{k} r_{b i}\right)=0 \\
& \gamma_{k}\left(\sum_{i=1}^{k} g\left(r_{t i}, r_{b i}\right)-\sum_{i=1}^{k} E_{s i}\right)=0 \\
& \theta_{1 i} r_{t i}=\theta_{2 i} r_{b i}=0, \quad \lambda_{1 k}, \lambda_{2 k}, \gamma_{k} \geq 0
\end{aligned}
$$

Taking the derivatives of $\mathcal{L}$ with respect to $r_{t i}$ and $r_{b i}$ :

$$
\begin{aligned}
-\sum_{k=i}^{N} \lambda_{1 k}+\left(\sum_{k=i}^{N} \gamma_{k}\right) \sigma_{1}^{2} 2^{2\left(r_{t i}+r_{b i}\right)}-\theta_{1 i} & =0 \\
-\sum_{k=i}^{N} \lambda_{2 k}+\left(\sum_{k=i}^{N} \gamma_{k}\right)\left(g\left(r_{t i}, r_{b i}\right)+\sigma_{2}^{2}\right)-\theta_{2 i} & =0
\end{aligned}
$$

From (31) and (32), we get:

$$
\begin{aligned}
g\left(r_{t i}, r_{b i}\right) & =\frac{\theta_{2 i}+\sum_{k=i}^{N} \lambda_{2 k}}{\sum_{k=i}^{N} \gamma_{k}}-\sigma_{2}^{2} \\
2^{2\left(r_{t i}+r_{b i}\right)} & =\frac{\theta_{1 i}+\sum_{k=i}^{N} \lambda_{1 k}}{\sigma_{1}^{2} \sum_{k=i}^{N} \gamma_{k}}
\end{aligned}
$$

Lemma 6 When the optimal total source power $g\left(r_{t i}^{*}, r_{b i}^{*}\right)$ increases, the energy buffer must be empty.

Proof: We will show that if $g\left(r_{t i}, r_{b i}\right)<g\left(r_{t, i+1}, r_{b, i+1}\right)$ then $\gamma_{i}>0$. First, assume $r_{b, i+1}>0$ which implies from (30) that $\theta_{2, i+1}=0$. Then, from (33), $g\left(r_{t i}, r_{b i}\right)<g\left(r_{t, i+1}, r_{b, i+1}\right)$ is only possible if $\gamma_{i}>0$. Next, assume $r_{b, i+1}=0$ which implies that $r_{t, i+1}>0$ otherwise $g\left(r_{t, i+1}, r_{b, i+1}\right)=0$ which cannot be optimal. When $r_{b, i+1}=0, g\left(r_{t i}, r_{b i}\right)<g\left(r_{t, i+1}, r_{b, i+1}\right)$ is equivalent to $2^{2\left(r_{t i}+r_{b i}\right)}<2^{2\left(r_{t, i+1}+r_{b, i+1}\right)}$, and from (34) and $\theta_{1, i+1}=0$, we must have $\gamma_{i}>0$.

Next, we show that the total source power cannot strictly decrease over the slots. 
Lemma 7 The total source power must be non-decreasing, i.e., $g\left(r_{t i}^{*}, r_{b i}^{*}\right) \leq g\left(r_{t, i+1}^{*}, r_{b, i+1}^{*}\right), \forall i$.

Proof: We will prove this statement by contradiction. Specifically, we assume a policy in which there is a slot $k$ such that $g\left(r_{t k}^{*}, r_{b k}^{*}\right)>g\left(r_{t, k+1}^{*}, r_{b, k+1}^{*}\right)$. We will show that this policy cannot be optimal.

We first show that if $g\left(r_{t k}, r_{b k}\right)>g\left(r_{t, k+1}, r_{b, k+1}\right)$ then $\lambda_{1 k}=\lambda_{2 k}=0$ cannot happen. First, assume $r_{b k}>0$ which implies from (30) that $\theta_{2 k}=0$. Then, from (33), $g\left(r_{t k}, r_{b k}\right)>g\left(r_{t, k+1}, r_{b, k+1}\right)$ is only possible if $\lambda_{2 k}>0$. Next, assume $r_{b k}=0$ which implies that $r_{t k}>0$ otherwise $g\left(r_{t k}, r_{b k}\right)=0$ which cannot be optimal. When $r_{b k}=0$, $g\left(r_{t k}, r_{b k}\right)>g\left(r_{t, k+1}, r_{b, k+1}\right)$ is equivalent to $2^{2\left(r_{t k}+r_{b k}\right)}>$ $2^{2\left(r_{t, k+1}+r_{b, k+1}\right)}$, and from (34) and $\theta_{1 k}=0$, we have $\lambda_{1 k}>0$.

Now, for $g\left(r_{t k}^{*}, r_{b k}^{*}\right)>g\left(r_{t, k+1}^{*}, r_{b, k+1}^{*}\right)$ to happen, we need to have either $r_{t k}^{*}>r_{t, k+1}^{*}, r_{b k}^{*} \leq r_{b, k+1}^{*}$ or $r_{b k}^{*}>$ $r_{b, k+1}^{*}, r_{t k}^{*} \leq r_{t, k+1}^{*}$ or $r_{t k}^{*}>r_{t, k+1}^{*}, r_{b k}^{*}>r_{b, k+1}^{*}$. We will examine these cases separately.

Case 1: $r_{t k}^{*}>r_{t, k+1}^{*}, r_{b k}^{*} \leq r_{b, k+1}^{*}$ : We must have $r_{t k}^{*}>0$ which implies $\theta_{1 k}=0$. In this case, for $g\left(r_{t k}^{*}, r_{b k}^{*}\right)>$ $g\left(r_{t, k+1}^{*}, r_{b, k+1}^{*}\right)$, we must also have $r_{t k}^{*}+r_{b k}^{*}>r_{t, k+1}^{*}+$ $r_{b, k+1}^{*}$. This implies from (34) that $\lambda_{1 k}>0$ and $\sum_{i=1}^{k} \bar{r}_{t i}^{*}=$ $\sum_{i=1}^{k} r_{t i}^{*}$. From the data causality constraints at the $(k-1) \mathrm{st}$ slot and $\sum_{i=1}^{k} \bar{r}_{t i}^{*}=\sum_{i=1}^{k} r_{t i}^{*}$, we must have $\bar{r}_{t k} \geq r_{t k}$. Similarly, from data causality at the $(k+1)$ st slot and $\sum_{i=1}^{k} \bar{r}_{t i}^{*}=\sum_{i=1}^{k} r_{t i}^{*}$, we must have $\bar{r}_{t, k+1} \leq r_{t, k+1}$. This implies that we must have $\bar{r}_{t k} \geq r_{t k}>r_{t, k+1} \geq \bar{r}_{t, k+1}$, thus $\bar{r}_{t k}>\bar{r}_{t, k+1}$. Now, consider the following modified policy for some $\delta>0, \hat{r}_{t k}=r_{t k}^{*}-\delta, \hat{r}_{t, k+1}=r_{t, k+1}^{*}+\delta, \hat{r}_{t k}=\bar{r}_{t k}^{*}-$ $\delta, \hat{r}_{t, k+1}=\bar{r}_{t, k+1}^{*}+\delta$. Data causality constraints are trivially satisfied. Energy causality at the top node can be satisfied by letting $\hat{P}_{t k}=\bar{P}_{t k}-\epsilon$ and $\hat{P}_{t, k+1}=\bar{P}_{t, k+1}+\epsilon$ because there exists $\epsilon>0$ such that $\bar{r}_{t k}^{*} \leq f\left(\left(1-\beta_{k}\right)\left(\bar{P}_{t k}-\epsilon\right) / \sigma_{3}^{2}\right)$ and $\bar{r}_{t, k+1}^{*} \leq f\left(\left(1-\beta_{k+1}\right)\left(\bar{P}_{t, k+1}+\epsilon\right) / \sigma_{3}^{2}\right)$. Energy causality at the source node is satisfied since at slot $k$ we have $g\left(r_{t k}^{*}-\delta, r_{b k}^{*}\right)<g\left(r_{t k}^{*}, r_{b k}^{*}\right)$ and at slot $k+1$ we have $g\left(r_{t k}^{*}-\right.$ $\left.\delta, r_{b k}^{*}\right)+g\left(r_{t, k+1}^{*}+\delta, r_{b, k+1}^{*}\right)<g\left(r_{t k}^{*}, r_{b k}^{*}\right)+g\left(r_{t, k+1}^{*}, r_{b, k+1}^{*}\right)$ due to joint convexity of $g(\cdot, \cdot)$ and $r_{t k}^{*}+r_{b k}^{*}>r_{t, k+1}^{*}+r_{b, k+1}^{*}$. This means that the modified policy is feasible, forwards the same amount of data, and consumes strictly less energy than the original one. This additional energy can be used to increase $r_{b k}^{*}$ and $r_{t k}^{*}$ which causes the data buffers at the top and bottom relays to be non-empty. This modified policy cannot be optimal because it does not satisfy the fact that if $g\left(r_{t k}, r_{b k}\right)$ strictly decreases in time, then both $\lambda_{1 k}$ and $\lambda_{2 k}$ cannot be zero, as proved at the beginning above. This also means the original policy cannot be optimal because its throughput is equal to the throughput of a sub-optimal policy.

Case 2: $r_{b k}^{*}>r_{b, k+1}^{*}, r_{t k}^{*} \leq r_{t, k+1}^{*}$ : We must have $r_{b k}^{*}>0$, therefore $\theta_{2 k}=0$. From $\lambda_{2 k}>0$, we must have that the bottom data buffer is empty, which implies $\sum_{i=1}^{k} \bar{r}_{b i}^{*}=\sum_{i=1}^{k} r_{b i}^{*}$. From this point on, the proof follows exactly as in Case 1 but with modifications to $r_{b k}^{*}, \bar{r}_{b k}^{*}, \bar{P}_{b k}$ instead of to $r_{t k}^{*}, r_{t k}^{*}, \bar{P}_{t k}$, and we conclude that this case cannot happen.
Case 3: $r_{b k}^{*}>r_{b, k+1}^{*}, r_{t k}^{*}>r_{t, k+1}^{*}$ : This case follows the same line of reasoning as the previous cases and by modifying both $r_{t k}^{*}, r_{b k}^{*}$ we reach to the same conclusion.

To summarize, since none of the above cases can be true, we have $g\left(r_{t i}^{*}, r_{b i}^{*}\right) \leq g\left(r_{t, i+1}^{*}, r_{b, i+1}^{*}\right), \forall i$.

We can always impose the constraint $\sum_{i=1}^{N} g\left(r_{t i}^{*}, r_{b i}^{*}\right)=$ $\sum_{i=1}^{N} E_{s i}$ on the problem in (13) because this does not change the optimal value. From Lemma 7, the total source power must be non-decreasing, and from Lemma 6 , the total source power can only increase when the energy buffer is empty. The source power policy that satisfies these properties is the unique singleuser optimal power policy [3], [5].

\section{REFERENCES}

[1] B. Schein and R. Gallager, "The Gaussian parallel relay network," in IEEE ISIT, June 2000.

[2] V. Prelov and E. van der Meulen, "Asymptotic expansion for the capacity region of the multiple-access channel with common information and almost Gaussian noise," in IEEE ISIT, June 1991.

[3] J. Yang and S. Ulukus, "Optimal packet scheduling in an energy harvesting communication system," IEEE Trans. Comm., vol. 60, pp. 220-230, January 2012.

[4] K. Tutuncuoglu and A. Yener, "Optimum transmission policies for battery limited energy harvesting nodes," IEEE Trans. Wireless Comm., vol. 11, pp. 1180-1189, March 2012.

[5] O. Ozel, K. Tutuncuoglu, J. Yang, S. Ulukus, and A. Yener, "Transmission with energy harvesting nodes in fading wireless channels: Optimal policies," IEEE JSAC, vol. 29, pp. 1732-1743, September 2011.

[6] J. Yang, O. Ozel, and S. Ulukus, "Broadcasting with an energy harvesting rechargeable transmitter," IEEE Trans. Wireless Comm., vol. 11, pp. 571-583, February 2012.

[7] M. A. Antepli, E. Uysal-Biyikoglu, and H. Erkal, "Optimal packet scheduling on an energy harvesting broadcast link," IEEE JSAC, vol. 29, pp. 1721-1731, September 2011.

[8] O. Ozel, J. Yang, and S. Ulukus, "Optimal broadcast scheduling for an energy harvesting rechargeable transmitter with a finite capacity battery," IEEE Trans. Wireless Comm., vol. 11, pp. 2193-2203, June 2012.

[9] J. Yang and S. Ulukus, "Optimal packet scheduling in a multiple access channel with energy harvesting transmitters," Journal of Comm. and Netw., vol. 14, pp. 140-150, April 2012.

[10] K. Tutuncuoglu and A. Yener, "Sum-rate optimal power policies for energy harvesting transmitters in an interference channel," Journal of Comm. and Netw., vol. 14, pp. 151-161, April 2012.

[11] B. Devillers and D. Gunduz, "A general framework for the optimization of energy harvesting communication systems with battery imperfections," Journal of Comm. and Netw., vol. 14, pp. 130-139, April 2012.

[12] K. Tutuncuoglu, A. Yener, and S. Ulukus, "Optimum policies for an energy harvesting transmitter under energy storage losses," IEEE JSAC, vol. 33, pp. 467-481, March 2015.

[13] O. Orhan, D. Gunduz, and E. Erkip, "Energy harvesting broadband communication systems with processing energy cost," IEEE Trans. Wireless Comm., vol. 13, pp. 6095-6107, November 2014.

[14] J. Xu and R. Zhang, "Throughput optimal policies for energy harvesting wireless transmitters with non-ideal circuit power," IEEE JSAC, vol. 32, pp. 322-332, February 2014.

[15] C. Huang, R. Zhang, and S. Cui, "Throughput maximization for the Gaussian relay channel with energy harvesting constraints," IEEE JSAC, vol. 31, pp. 1469-1479, August 2013.

[16] D. Gunduz and B. Devillers, "Two-hop communication with energy harvesting," in IEEE CAMSAP, December 2011.

[17] O. Orhan and E. Erkip, "Optimal transmission policies for energy harvesting two-hop networks," in CISS, March 2012.

[18] Y. Luo, J. Zhang, and K. B. Letaief, "Optimal scheduling and power allocation for two-hop energy harvesting communication systems," IEEE Trans. Wireless Comm., vol. 12, pp. 4729-4741, September 2013.

[19] O. Orhan and E. Erkip, "Throughput maximization for energy harvesting two-hop networks," in IEEE ISIT, July 2013.

[20] B. Gurakan and S. Ulukus, "Energy harvesting diamond channel with energy cooperation," in IEEE ISIT, June 2014. 